\title{
Association Between Anxious Symptom Severity and Olfactory Impairment in Young Adults with Generalized Anxiety Disorder: A Case-Control Study
}

\author{
Xiacan Chen' \\ Wanjun Guo ${ }^{2}$ \\ Liangming $\mathrm{Yu}^{2}$ \\ Dan Luo ${ }^{2}$ \\ Leling $\mathrm{Xie}^{2}$ \\ Jiajun $\mathrm{Xu} \mathbb{1}^{2}$
}

'Institute of Forensic Medicine, West China School of Basic Medical Sciences \& Forensic Medicine, Sichuan University, Chengdu, People's Republic of China; ${ }^{2}$ Mental Health Centre, West China Hospital, Sichuan University, Chengdu, People's Republic of China
Correspondence: Jiajun $\mathrm{Xu}$ Mental Health Centre, West China Hospital, Sichuan University, 61004I, People's Republic of China Email xujiajun 120@I26.com
Objective: The findings of olfaction are inconsistent in anxiety disorders, and few studies have reported on the olfactory performance in generalized anxiety disorder (GAD). Therefore, we aim to investigate the olfactory function of patients with GAD and the association between olfactory function and anxiety symptoms.

Methods: We conducted a case-control study by selecting 107 patients with GAD and 107 healthy controls matched for sex and age, to investigate olfaction deficits in GAD and the association between anxiety severity and olfaction. All patients were treatment-naïve prior to the inclusion. Anxiety severity and olfaction were assessed by the Hamilton anxiety rating scale (HAM-A) and the Sniffin' Sticks test (SST), respectively. Partial correlations were used to analyze the relationship between olfaction and anxiety severity. False discovery rate (FDR) correction was used in multi-correlation analyses.

Results: Compared with healthy controls, patients in the GAD group demonstrated odor threshold, discrimination and identification deficits. In the moderate/severe anxiety subgroup, discrimination score was significantly and inversely correlated with the somatic anxiety score $(\gamma=-0.44, q=0.03)$, and identification score was significantly and inversely correlated with the HAM-A total score $(\gamma=-0.42, \mathrm{q}=0.04)$. The TDI score (the sum of threshold, discrimination and identification scores) was significantly and inversely correlated with the somatic $(\gamma=$ $-0.44, \mathrm{q}=0.04)$ and psychic $(\gamma=-0.45, \mathrm{q}=0.04)$ anxiety scores in the moderate/severe anxiety subgroup.

Conclusion: This study demonstrated the olfactory impairment in patients with GAD and that poorer odor discrimination was correlated with greater severity of somatic anxiety.

Keywords: GAD, olfaction, symptom

\section{Introduction}

Generalized anxiety disorder (GAD) includes persistent and excessive anxiety and worry with apprehensive expectations of events or activities. In addition, patients present with physical symptoms, including feeling keyed up or on edge, becoming easily fatigued and having difficulty concentrating. ${ }^{1}$

Previous studies have reported that the emotional dysregulation of GAD is related to the hyperresponsiveness of the amygdala ${ }^{2}$ and a less functional connectivity between the amygdala and prefrontal frontal cortex. ${ }^{3}$ Interestingly, part of the amygdala, particularly the cortical amygdala nuclei, and other cerebral structures (such as 
the piriform cortex and a small part of the anterior parahippocampal gyrus) form the primary olfactory cortex. ${ }^{1,4}$ There is only one synapse between the primary olfactory cortex and the olfactory receptor, making olfactory function one of the most direct sensory connections between the external environment and the central nervous system. ${ }^{1}$ Considering these factors, the olfactory studies may provide insight into understanding the anxiety symptoms in GAD.

Mounting evidence has indicated that olfaction, including threshold, discrimination and identification functions, is related to emotional dysfunction. A lowered odor threshold was reported in patients with bipolar disorder, ${ }^{5}$ and poor odor sensitivity and odor identification have been reported in patients with depression. ${ }^{6,7}$ Segalas et al reported olfactory impairment in patients with obsessive-compulsive disorder (OCD) and that odor identification was correlated with the severity of OCD symptoms. ${ }^{8}$ A few studies have investigated the relationship between anxiety disorders and olfaction. Burón et al reported a lower odor threshold in patients with panic disorder and that the symptom severity of panic disorder was correlated with olfactory questionnaire ratings, ${ }^{9}$ and Clepce et al found poor odor discrimination in anxiety disorders. ${ }^{10}$ However, olfaction in GAD has not been well studied. Sex, age and smoking have been reported as confounding factors in previous studies of olfaction conducted in the general population or in those with psychotic disorders, ${ }^{11-14}$ making the mechanisms of the relationship between olfaction and GAD difficult to determine.

Being female is considered as a risk factor of GAD, and women are found to be less likely to relapse, ${ }^{15,16}$ while men are more predisposed to somatic symptoms. ${ }^{17}$ Moreover, sex has played an important role in olfactory function, ${ }^{18,19}$ making it necessary to explore sex differences in olfaction in GAD patients.
Given these previous studies, we hypothesized that the patients with GAD might present olfactory impairment. To test our hypothesis, we conducted a case-control study matched by sex and age to compare the olfaction between patients with GAD and healthy controls to investigate olfactory function of patients with GAD, as well as the association between the olfactory function and symptoms of anxiety.

\section{Methods}

\section{Procedure and Participants}

We recruited 107 first-time patients diagnosed with GAD (47 men, 60 women) (Table 1), and all patients were treatment-naïve prior to the inclusion. Young adults (18-35 years old) were selected as participants because age is an important confounder of olfaction and olfaction differs between young (18-35 years old), middle-aged and older adults. ${ }^{12,13}$ The Structured Clinical Interview for DSM-IV (SCID-I/P) was conducted by a psychiatric resident doctor for the diagnostic assessment, and GAD was ultimately diagnosed by face-to-face interviews by two senior psychiatrists according to DSM-IV. Because SCID-I/P is a structural assessment under the framework of DSM-IV, we chose DSM-IV as the diagnostic criteria in this study. Other mental disorders were excluded by SCID-I/P. Organic brain injury, neurological disorder, any history of major olfactory disturbance (eg, nasal congestion, rhinitis), other mental disorders, and poor physical health were excluded by using a self-administered questionnaire. Participants were assessed before receiving pharmacological treatment.

We recruited 107 healthy controls by advertising. They were age- ( \pm 3 years $)$ and sex-matched with GAD patients and screened by the SCID-I/P to exclude any mental disorders. Individuals were excluded if there was a family history of mental disorder among immediate relatives or if

Table I Demographic Characteristics, Anxiety Status and Olfactory Performance Scores

\begin{tabular}{|c|c|c|c|c|}
\hline & GAD Group & Healthy Control Group & & $P$ value \\
\hline & $N=107$ & $N=107$ & & \\
\hline Age (years) & $26.68(5.79)$ & $26.08(4.08)$ & $t=-0.87$ & 0.38 \\
\hline Sex, male(n) & 47 & 47 & & \\
\hline Smoking, $n(\%)$ & $32(29.9)$ & $10(9.3)$ & $\chi^{2=}=14.34$ & 0.002 \\
\hline HAM-A, mean (SD) in score & & & & \\
\hline Somatic anxiety score & $5.58(5.60)$ & I & l & \\
\hline Psychic anxiety score & I2.68(4.84) & l & l & \\
\hline Total score & 18.26(9.72) & l & I & \\
\hline
\end{tabular}

Abbreviations: GAD, generalized anxiety disorder; SST, the Sniffin' Sticks test; TDI, The total score of threshold, discrimination and identification. 
they had organic brain injury, neurological disorder, any history of major olfactory disturbance (eg, nasal congestion and rhinitis), other mental disorders, or poor physical health. This study was approved by the Ethical Review Board of the West China Hospital (N0. 2018-109), Sichuan University. All healthy participants and patients provided written informed consent.

\section{Measures}

\section{Demographic Characteristics}

Demographic characteristics, including age, sex, smoking status, any history of major olfactory disturbance, and participants' health status and disease history, including problems of olfactory system, were assessed in the face-toface interview using a self-report questionnaire.

\section{General Anxiety Disorder Symptom Assessment}

The Hamilton anxiety rating scale (HAM-A) contains 14 items with a 4-point rating for each item and was used to assess anxious symptom severity. ${ }^{20,21}$ The instrument consists of somatic anxiety (SA) and psychic anxiety (PA) sub-scales. The Chinese version of HAM-A was used to evaluate anxious symptom in this study, which has good reliability of $\gamma=0.93$ and good interrater reliability (three raters) of 0.91 . The Moderate/severe anxiety is indicated by a total score of $>21$, while the minor levels of anxiety are indicated by a total score of $\leq 21) .^{22,23}$

\section{Olfactory Testing}

The Sniffin' Sticks test, with high repeatability, was used to evaluate olfaction in this study. ${ }^{11,24}$ It is composed of threshold, discrimination and identification odor tests. The odor threshold task consists of 16 pens with different concentrations of n-butanol solution. The concentrations range from $4 \%$ (designated as pen no. 1) to $1.2 \mathrm{ppmv}$ (parts per million, by volume) (pen no. 16). ${ }^{25}$ The triplet of felt-tipped pens is a group of two containing blank solvent and a third containing the odorant of n-butanol solution. The interval between the presentation of each pen and each group was approximately 3 and 30 seconds, respectively. The triplets are presented to the subject in randomized order, and the subject is asked to determine the odorant from the other two blank solvents.

The odor discrimination task also comprises 16 groups. A group consisting of a triplet of pens, includes two with the same odorant and a third with a different odorant. The interval between the presentation of each pen and each group was approximately 3 and 30 seconds, respectively. A triplet of pens is randomly presented to the subject, and the subject must identify which one smells differently.

During the odor identification test, 16 odors were presented to participants through the felt-tipped pen dispensers, and participants were asked to identify each odor from a list of four descriptors. The interval between the presentation of each pen was approximately 3 seconds.

The score of each sub-test ranges from 0 to 16 , and the TDI total score (the sum of threshold, discrimination, and identification scores) ranges from 0 to 48 . Lower SST scores indicate worse olfaction.

\section{Statistical Analyses}

The normal distributions of data were confirmed by normal P-P plot and detrended normal P-P plot. Age, the HAM-A scores, and the SST scores followed an approximately normal distribution. Therefore, mean $( \pm \mathrm{SD})$ was used to describe continuous variables and a $t$-test was used to compare continuous variables between groups. The chi-square test was used to compare categorical variables between two groups. The GAD group was further divided into two subgroups - a moderate/severe anxiety level group (HAM-A total score $>21$ ), and a minor anxiety level group (HAM-A total score $\leq 21$ ). To control for smoking status in the correlation analysis, we chose partial correlation analysis to show the relationship between clinical scales (HAM-A) and the SST scores in the GAD group, moderate/severe anxiety subgroup, and male and female subgroups. False discovery rate (FDR) correction was used in multi-correlation analyses and $\mathrm{q}$ was used to indicate an FDR-corrected $p$ value. An alpha level of $p<0.05$ or $q<0.05$ was defined as statistically significant. All data analyses were performed with the statistical package SPSS (version 16.0).

\section{Results}

The age, sex and smoking status of the GAD and healthy groups are listed in Table 1, as well as the HAM-A scores of GAD group. The proportion of smokers in the GAD group was more than three times that of the healthy group (Table 1).

Compared with the healthy group, the GAD group had significantly poorer odor sensitivity (threshold score: GAD = $6.13 \pm 2.80$, Healthy $=8.48 \pm 3.04, t=5.88, \mathrm{p}<0.01)$, discrimination (discrimination score: $\mathrm{GAD}=10.27 \pm 2.88$, Healthy $=12.23 \pm 1.96, t=5.83, \mathrm{p}<0.01)$ and identification (identification score: $\mathrm{GAD}=11.41 \pm 2.51$, Healthy $=12.92 \pm$ 


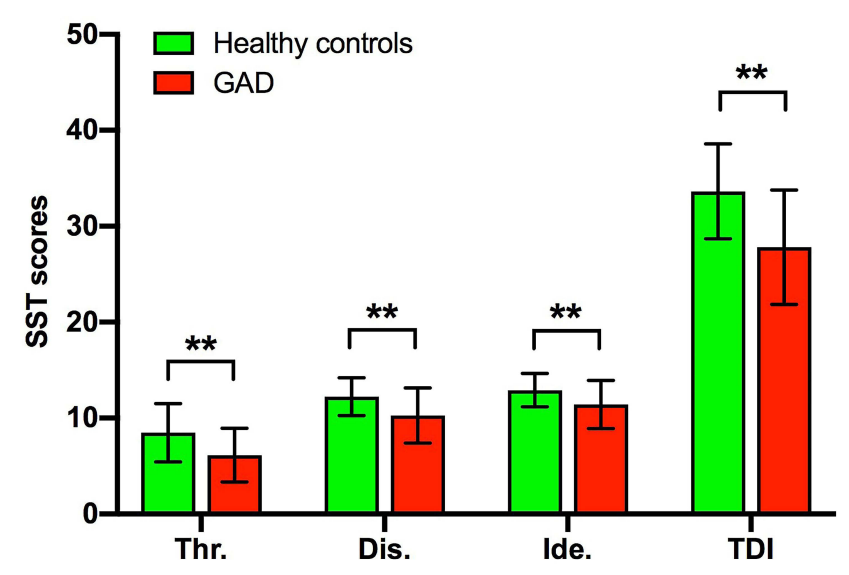

Figure I The difference of SST scores between the GAD and healthy groups. **p $<$ 0.01 .

Abbreviations: GAD, generalized anxiety disorder; Thr., threshold; Dis., discrimination; Ide., identification; SST: Sniffin' Sticks test; TDI, the total score of threshold, discrimination and identification.

1.73, $t=5.10, \mathrm{p}<0.01$ ) (Figure 1 ), as well as a significantly lower TDI score $(\mathrm{GAD}=27.81 \pm 5.97$, Healthy $=33.63 \pm 4.94$, $t=7.76, \mathrm{p}<0.01$ ) (Figure 1). We found no significant correlation between anxiety symptoms and olfactory performance in the total GAD group, nor in the male and female subgroups of GAD while controlling for smoking status (Figure 2).

The minor anxiety level subgroup included 37 men and 39 women and the moderate/severe anxiety subgroup included 10 men and 21 women. The mean scores of the
SA subscale, PA subscale, and HAM-A total score of anxiety level subgroups are listed in Table 2 . The differences of odor threshold, discrimination, identification and TDI total scores did not differ significantly between patients with moderate/severe anxiety level and patients with minor anxiety level (Table 2).

A heat map of partial correlation coefficients between the SST score and HAM-A score in GAD while controlling for smoking status is presented in Figure 2. In the minor anxiety level group, SST score were not significantly correlated with the HAM-A score under the control of smoking $(q>0.05)$. In the moderate/severe anxiety level subgroup, the SA score was inversely and significantly correlated with the discrimination $(\gamma=-0.44, \mathrm{q}=0.03)$ and TDI total $(\gamma=-0.44, \mathrm{q}=$ $0.04)$ scores; the PA score was inversely and significantly correlated with the TDI total score $(\gamma=-0.45, q=0.04)$. The HAM-A total score was inversely and significantly correlated with the identification score $(\gamma=-0.42, \mathrm{q}=0.04)$.

In the GAD group, the odor threshold, discrimination, identification, and TDI total scores did not differ significantly between male and female GAD patients, nor did the SA, PA, and HAM-A scores (Table 2). The SST scores were not significantly correlated with the HAM-A scores either in the male GAD or in the female GAD subgroup (Figure 2).
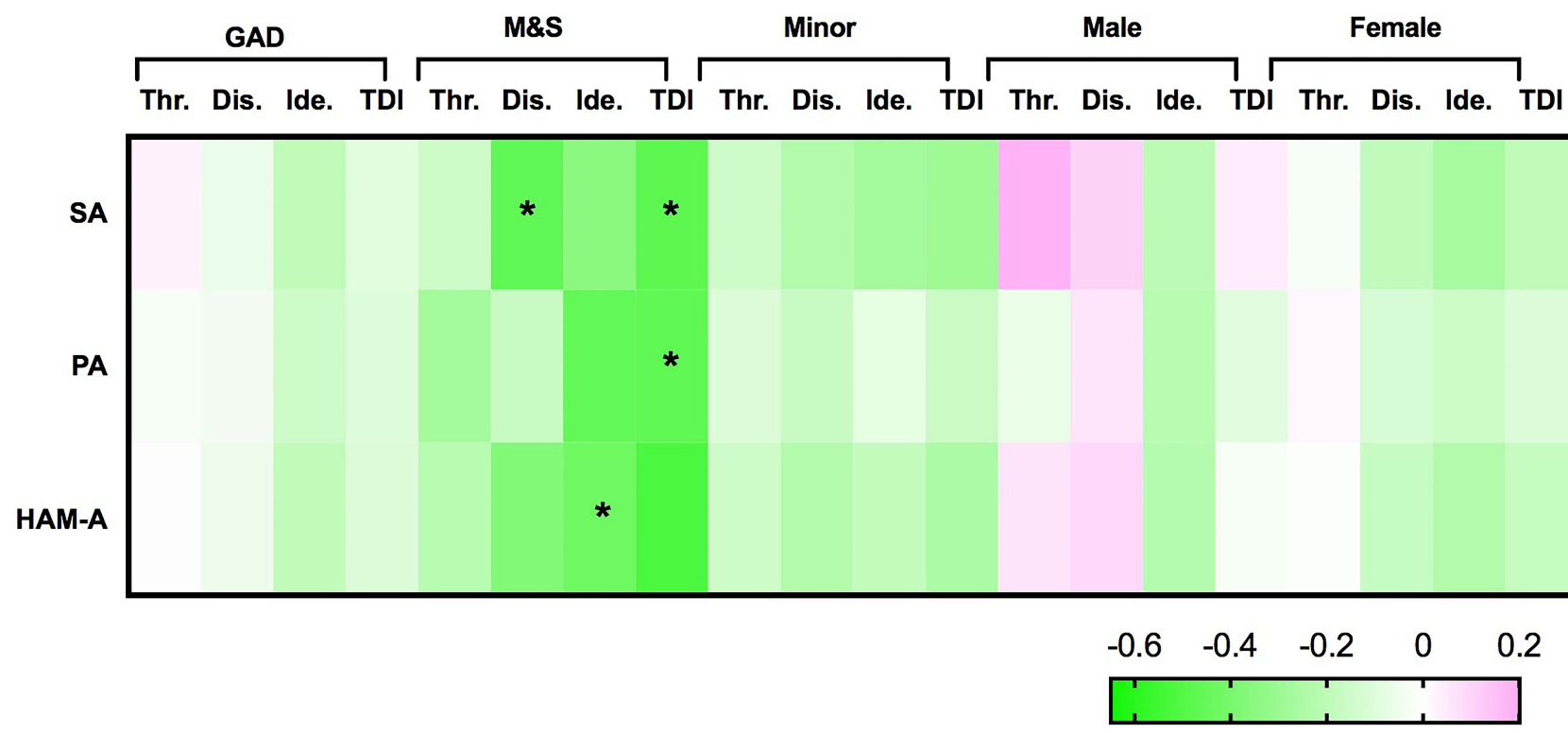

Figure 2 Heat map of partial correlation coefficients between SST scores and HAM-A scores in the GAD group while controlling smoking status. The pink box indicates a positive correlation; the green box indicates a negative correlation. The brightness of each box is proportional to the magnitude of correlation coefficient. $* q<0.05$. Abbreviations: $q$, FDR-corrected $p$ value; GAD, generalized anxiety disorder; M\&S, Moderate/severe GAD subgroup (HAM-A >2I); Minor, minor GAD subgroup (HAM-A $\leq 21$ ); Male, male GAD subgroup; Female, female GAD subgroup; HAM-A, Hamilton anxiety rating scale; TDI, the total score of threshold, discrimination and identification. 
Table 2 The Comparison of HAM-A Scores and SST Scores Between Severity and Sex Subgroups of GAD

\begin{tabular}{|c|c|c|c|c|c|c|c|c|}
\hline & \multicolumn{4}{|c|}{ Severity Subgroups } & \multicolumn{4}{|c|}{ Sex Subgroups } \\
\hline & Minor/Lower $n=76$ & Moderate/Severe $n=3$ I & $t$ & $p$ value & Male & Female & $t$ & $p$ value \\
\hline HAM-A scores & & & & & & & & \\
\hline Somatic anxiety & $2.8(2.59)$ & $12.32(5.28)$ & -12.46 & $<0.01$ & $4.83 \pm 5.33$ & $6.17 \pm 5.78$ & -1.23 & 0.22 \\
\hline Psychic anxiety & $10.7(3.77)$ & $17.48(3.70)$ & -8.46 & $<0.01$ & $11.70 \pm 5.00$ & $13.45 \pm 4.60$ & -1.88 & 0.06 \\
\hline Total score & I $3.6(5.37)$ & $29.81(8.25)$ & -12.04 & $<0.01$ & $16.53 \pm 9.67$ & $19.62 \pm 9.63$ & -1.64 & 0.10 \\
\hline SST scores & & & & & & & & \\
\hline Threshold & $5.89(2.78)$ & $6.71(2.80)$ & -1.37 & 0.17 & $6.21 \pm 2.40$ & $6.07 \pm 3.09$ & 0.27 & 0.79 \\
\hline Discrimination & $10.05(3.10)$ & $10.8 \mathrm{I}(2.2 \mathrm{I})$ & -1.23 & 0.22 & $10.30 \pm 2.94$ & $10.25 \pm 2.86$ & 0.09 & 0.93 \\
\hline Identification & II.46 (2.47) & II.32 (2.57) & 0.23 & 0.82 & $11.36 \pm 2.47$ & $11.45 \pm 2.57$ & -0.18 & 0.86 \\
\hline TDI & $27.39(6.32)$ & $28.84(4.97)$ & -1.14 & 0.26 & $27.87 \pm 5.56$ & $27.77 \pm 6.32$ & 0.09 & 0.93 \\
\hline
\end{tabular}

Abbreviations: GAD, generalized anxiety disorder; SST, the Sniffin' Sticks test; TDI, the total score of threshold, discrimination and identification.

\section{Discussion}

We demonstrated that compared with healthy controls, patients with GAD have impaired olfaction, including threshold, discrimination, and identification impairment. Lower SST scores were associated with worse the olfactory function. Our results further showed that the degree of odor discrimination impairment was positively correlated with somatic anxiety severity, and the degree of odor identification impairment was positively correlated with anxiety severity in the subgroup of GAD patients with moderate/severe anxiety symptoms while controlling for smoking status. Sex differences were not significant in the relationship between the olfactory impairment and anxiety severity.

In contrast to the established olfactory deficit found in patients with psychotic symptoms, the findings of olfaction are inconsistent among individuals with anxiety disorders. We found odor threshold impairment among $\mathrm{GAD}$, which is consistent with prior research that also reported impaired odor threshold in panic disorder. ${ }^{9}$ Our result of odor discrimination deficit results correspond to a previous study reporting poor odor discrimination among patients with social phobia, panic disorder, agoraphobia and GAD, ${ }^{10}$ whereas another study did not find a significant difference between healthy controls and anxiety patients. ${ }^{26}$ It has been argued that these inconsistent findings are due to the different olfaction tests used by different researchers; ${ }^{27}$ however, we tend to think that different key symptoms of different anxiety disorders are the main factors accounting for these conflicting findings. In contrast to the inconsistent results of olfactory impairment in anxiety disorders, olfactory studies investigating psychotic disorders also used different olfactory test tools and showed consistent reports of odor identification impairment. ${ }^{28}$ It is therefore possible that it is the result of different key symptoms, rather than different olfactory tests, that can explain the conflicting findings in previous studies. Further olfactory studies in terms of anxiety disorders are needed and should focus on key symptoms.

After controlling for smoking status, we found that the severity of somatic anxiety symptoms was positively correlated with the odor discrimination impairment in patients with moderate/severe levels of anxiety severity. This finding corresponds to that the olfactory and affective processes overlap in the cerebral anatomy structure-the cortical amygdala nuclei. ${ }^{29,30}$ The amygdala and the orbitofrontal cortex are the primary implicated neurocircuitries of anxiety disorders. ${ }^{31}$ The primary olfactory cortex, including the cortical amygdala nuclei, transports odor information to other parts of the amygdala. ${ }^{1,4}$ Dysfunction in the overlapping structure - the amygdala - may therefore be the crucial factor in the relationship.

Furthermore, the odor threshold is a more peripheral sensory process, which is related to olfactory receptor and odor molecule synapse. ${ }^{32}$ Odor discrimination and identification are central sensory processes that are related to the primary olfactory cortex. ${ }^{1}$ We speculate that this is why odor discrimination and identification impairments were related to anxiety symptom severity, whereas odor threshold was not. However, these relationships were only found in GAD patients with moderate/ severe level of anxiety severity. Therefore, this finding suggests that it is important to consider anxiety severity level when investigating the relationship between GAD symptoms and olfaction. 
Sex differences were not present in the relationship between olfaction and anxiety symptoms in this study, but mounting evidence has demonstrated such differences in the relationship between olfactory impairment and psychotic symptoms. ${ }^{14,33}$ However, we did not find other reports of sex differences regarding the relationship between GAD and olfaction. It is therefore crucial to conduct future studies exploring the olfaction in patients with GAD with consideration to potential sex differences.

This study also has some limitations. First, our results of showing relationship cannot determine the exact alterations in brain structure corresponding to the relationship between odor deficits and anxiety severity. Therefore, following research should include neuroimaging or electrophysiological data to search for a definitive underlying mechanism. Second, comparison of olfaction pre- and post-treatment may provide more useful information when interpreting olfactory impairment in GAD. These two points might be the direction of future studies. Finally, more nuanced descriptions of smoking status, such as how many cigarettes constitute smoking, should be investigated.

Our results revealed odor threshold, discrimination and identification deficits in GAD. Somatic anxiety was correlated with odor discrimination impairment and anxiety severity was correlated with identification impairment in patients with moderate or higher level of anxiety severity, whereas odor threshold was not correlated with anxiety severity. Further research should investigate the sensitivity of olfaction corresponding to anxiety treatment effects and the change in brain function related to olfactory alteration.

\section{Ethics Approval}

This study was conducted in accordance with the Declaration of Helsinki and approved by the Ethical Review Board of the West China Hospital (No. 2018109), Sichuan University. All healthy participants and patients provided written informed consent.

\section{Author Contributions}

All authors made substantial contributions to conception and design, acquisition of data, or analysis and interpretation of data; took part in drafting the article or revising it critically for important intellectual content; agreed to submit to the current journal; gave final approval of the version to be published; and agree to be accountable for all aspects of the work.

\section{Funding}

This study was supported by the Key Research and Development Project of Science and Technology Department, Sichuan Province (Grant no. 2019YFS0218); the Technology Innovation Research \& Development Project of Chengdu Science and Technology Bureau (Grant no. 2019-YF05-00240-SN); the China Postdoctoral Science Foundation (Grant no. 2020TQ0219); the Fundamental Research Funds for the Central Universities (Grant no. 2021SCU12080).

\section{Disclosure}

The authors have no potential conflicts of interest to disclose.

\section{References}

1. Nguyen AD, Shenton ME, Levitt JJ. Olfactory dysfunction in schizophrenia: a review of neuroanatomy and psychophysiological measurements. Harv Rev Psychiatry. 2010;18(5):279-292. doi:10.3109/10673229.2010.511060

2. Fitzgerald JM, Phan KL, Kennedy AE, et al. Prefrontal and amygdala engagement during emotional reactivity and regulation in generalized anxiety disorder. $J$ Affect Disord. 2017;218:398-406. doi:10.1016/j. jad.2017.05.013

3. Fitzgerald JM, Klumpp H, Langenecker S, et al. Transdiagnostic neural correlates of volitional emotion regulation in anxiety and depression. Depress Anxiety. 2019;36(5):453-464. doi:10.1002/da.22859

4. Nolte J. The Human Brain: An Introduction to Its Functional Anatomy. 6th ed. Mosby: Philadelphia; 2008:330-339.

5. Kruger S, Frasnelli J, Bräunig P, Hummel T. Increased olfactory sensitivity in euthymic patients with bipolar disorder with event-related episodes compared with patients with bipolar disorder without such episodes. J Psychiatry Neurosci. 2006;31(4):263-270.

6. Lombion-Pouthier S, Vandel P, Nezelof S, et al. Odor perception in patients with mood disorders. J Affect Disord. 2006;90(23):187-191. doi:10.1016/j.jad.2005.11.012

7. Clepce M, Gossler A, Reich K, et al. The relation between depression, anhedonia and olfactory hedonic estimates-a pilot study in major depression. Neurosci Lett. 2010;471(3):139-143. doi:10.1016/ j.neulet.2010.01.027

8. Segalas C, Labad J, Alonso P, et al. Olfactory identification and discrimination in obsessive-compulsive disorder. Depress Anxiety. 2011;28(10):932-940. doi:10.1002/da.20836

9. Burón E, Bulbena A, Bulbena-Cabré A. Olfactory functioning in panic disorder. J Affect Disord. 2015;175:292-298. doi:10.1016/j. jad.2015.01.049

10. Clepce M, Reich K, Gossler A, et al. Olfactory abnormalities in anxiety disorders. Neurosci Lett. 2012;511(1):43-46. doi:10.1016/j. neulet.2012.01.034

11. Kobal G, Hummel TH, Sekinger B, Barz S, Roscher S, Wolf S. "Sniffin' sticks": screening of olfactory performance. Rhinology. 1996;34(4):222-226.

12. Hummel T, Kobal G, Gudziol H, et al. Normative data for the "Sniffin' Sticks" including tests of odor identification, odor discrimination, and olfactory thresholds: an upgrade based on a group of more than 3000 subjects. Eur Arch Oto-Rhino-Laryngol. 2007;264 (3):237-243. doi:10.1007/s00405-006-0173-0 
13. Sorokowska A, Schriever VA, Gudziol V, et al. Changes of olfactory abilities in relation to age: odor identification in more than 1400 people aged 4 to 80 years. Eur Arch Oto-Rhino-Laryngol. 2015;272 (8):1937-1944. doi:10.1007/s00405-014-3263-4

14. Chen $\mathrm{X}, \mathrm{Xu} \mathrm{J}, \mathrm{Li} \mathrm{B}$, et al. Olfactory impairment in first-episode schizophrenia: a case-control study, and sex dimorphism in the relationship between olfactory impairment and psychotic symptoms. BMC Psychiatry. 2018;18(1):199. doi:10.1186/s12888-018-1786-8

15. Weisberg RB. Overview of generalized anxiety disorder: epidemiology, presentation, and course. J Clin Psychiatry. 2009;70(Suppl 2):4-9. doi:10.4088/JCP.s.7002.01

16. Moreno-Peral P, Conejo-Cerón S, Motrico E, et al. Risk factors for the onset of panic and generalised anxiety disorders in the general adult population: a systematic review of cohort studies. J Affect Disord. 2014;168:337-348. doi:10.1016/j.jad.2014.06.021

17. Boehlen FH, Herzog W, Schellberg D, et al. Gender-specific predictors of generalized anxiety disorder symptoms in older adults: results of a large population-based study. $J$ Affect Disord. 2020;262:174-181. doi:10.1016/j.jad.2019.10.025

18. Oliveira-Pinto AV, Santos RM, Coutinho RA, et al. Sexual dimorphism in the human olfactory bulb: females have more neurons and glial cells than males. PLoS One. 2014;9(11):e111733. doi:10.1371/ journal.pone.0111733

19. Khil L, Rahe C, Wellmann J, et al. Association between major depressive disorder and odor identification impairment. $J$ Affect Disord. 2016;203:332-338. doi:10.1016/j.jad.2016.06.022

20. Hamilton M. Diagnosis and rating of anxiety. $\mathrm{Br} J$ Med Psychol. 1969;3(special issue):76-79.

21. Hamilton M. The assessment of anxiety states by rating. $\mathrm{Br} \mathrm{J} \mathrm{Med}$ Psychol. 1959;32(1):50-55. doi:10.1111/j.2044-8341.1959.tb00467.x

22. Tang L. The Hamilton Anxiety Scale. Shanghai Arch Psychiatry. 19842:64-65.

23. Guoxian L, et al. The application of the Hamilton anxiety rating scale in neurosis. Chin J Neurol Psychiatry. 1986;19(6):342-344.
24. Kobal G, Klimek L, Wolfensberger M, et al. Multicenter investigation of 1036 subjects using a standardized method for the assessment of olfactory function combining tests of odor identification, odor discrimination, and olfactory thresholds. Eur Arch Oto-RhinoLaryngol. 2000;257(4):205-211. doi:10.1007/s004050050223

25. Hummel T, Sekinger B, Wolf SR, et al. 'Sniffin' sticks': olfactory performance assessed by the combined testing of odor identification, odor discrimination and olfactory threshold. Chem Senses. 1997;22 (1):39-52. doi:10.1093/chemse/22.1.39

26. Kamath V, Paksarian D, Cui L, et al. Olfactory processing in bipolar disorder, major depression, and anxiety. Bipolar Disord. 2018;20 (6):547-555. doi:10.1111/bdi.12625

27. Buron E, Bulbena A. Olfaction in affective and anxiety disorders: a review of the literature. Psychopathology. 2013;46(2):63-74. doi: $10.1159 / 000338717$

28. Houghton DC, Howard SL, Uhde TW, et al. Odor sensitivity impairment: a behavioral marker of psychological distress? CNS Spectr. 2019;24(4):404-412. doi:10.1017/S1092852918001177

29. Good KP, Sullivan RL. Olfactory function in psychotic disorders: insights from neuroimaging studies. World J Psychiatry. 2015;5 (2):210-221. doi:10.5498/wjp.v5.i2.210

30. Roy AK, Shehzad Z, Margulies DS, et al. Functional connectivity of the human amygdala using resting state fMRI. Neuroimage. 2009;45 (2):614-626. doi:10.1016/j.neuroimage.2008.11.030

31. Milad MR, Rauch SL. The role of the orbitofrontal cortex in anxiety disorders. Ann N Y Acad Sci. 2010;1121(1):546-561. doi:10.1196/ annals. 1401.006

32. Rupp CI, Fleischhacker WW, Kemmler G, et al. Various bilateral olfactory deficits in male patients with schizophrenia. Schizophr Bull. 2005;31(1):155-165. doi:10.1093/schbul/sbi018

33. Malaspina D, Goetz R, Keller A, et al. Olfactory processing, sex effects and heterogeneity in schizophrenia. Schizophr Res. 2012;135 (1-3):144-151. doi:10.1016/j.schres.2011.11.025
Neuropsychiatric Disease and Treatment

\section{Publish your work in this journal}

Neuropsychiatric Disease and Treatment is an international, peerreviewed journal of clinical therapeutics and pharmacology focusing on concise rapid reporting of clinical or pre-clinical studies on a range of neuropsychiatric and neurological disorders. This journal is indexed on PubMed Central, the 'PsycINFO' database and CAS, and is the official journal of The International Neuropsychiatric Association (INA). The manuscript management system is completely online and includes a very quick and fair peer-review system, which is all easy to use. Visit http://www.dovepress.com/testimonials.php to read real quotes from published authors. 